\title{
Differential Display of Protein Tyrosine Kinase cDNAs from Human Fetal and Adult Brains
}

BioTechniques 32:856-865 (April 2002)

\author{
M. Okada, S. Yamaga ${ }^{1}$, \\ S. Yasuda, S.M. Weissman', \\ and Y. Yasukochi \\ Tokyo Medical and Dental \\ University, Tokyo, Japan, and \\ ${ }^{1}$ Yale University School of Med- \\ icine, New Haven, CT, USA
}

\begin{abstract}
Here we describe a differential display method for surveying the expression of most protein tyrosine kinases and applying it to cDNAs from human fetal and adult brains. The method involves two selective steps for processing the mRNA. At each step, degenerate oligonucleotide primers derived from highly conserved regions of the catalytic domain of the kinases are used. In the display with $\mathrm{Bst} Y I$ and $\mathrm{BsiHKAI}$ digests of the cDNA, $65 \%$ and $59 \%$ of a total of 72 and 63 bands, respectively, represented fragments from a total of 27 different tyrosine kinases. The expression levels of the kinases in the display were comparable with those measured by RT-PCR. This method offers a relatively specific way to display differentially expressed gene families in any tissue and cell type.
\end{abstract}

\section{INTRODUCTION}

Protein tyrosine kinases play a pivotal role in cell functions, such as cell growth, differentiation, apoptosis, and carcinogenesis in a variety of cell types. During brain development, tyrosine kinases are involved in the formation of projection maps in the central nervous system $(1,4)$ and the promotion of neuron survival (1). For example, the Eph family of tyrosine kinase receptor and ligands, ephrins, direct the guidance of retinal, cortical, and hippocampal axons during development.

Although the cloning of protein tyrosine kinase has been performed using methods based on kinase-specific PCR amplification from cDNA libraries (10, 13), screening cDNA libraries with kinase-specific degenerate oligonucleotides (5), or with antibody against phosphorylated tyrosine residue (8), only one report is available concerning the cDNA display of the protein tyrosine kinase profile (6). In this report, the cDNA fragments were amplified by PCR using DFG and DVW primers derived from conserved regions of the tyrosine kinase catalytic domain and displayed on gels, following digestion with a restriction enzyme. The cDNA fragments amplified from the tyrosine kinases are all approximately 143 bp long so that the fragments from kinase cDNAs that lack a cleavage site in this segment would all run together on a sequencing gel. In addition, treatment with the enzyme shortens the fragments and may limit available information of their DNA sequences.

We developed an alternative method to display the protein tyrosine kinase cDNAs from fetal and adult brains that will generate discrete fragments of differing sizes from different tyrosine kinase mRNAs. We used this method to detect a total of 27 different protein kinases in the human brain.

\section{MATERIALS AND METHODS}

Poly(A)+ RNAs prepared from human fetal (a pool of 10 normal tissue specimens from 21-30-week gestation fetuses) and adult brains (a pool of normal tissue specimens from 43- and 55year-old individuals) were purchased from BD Biosciences Clontech (Palo Alto, CA, USA).

\section{cDNA Synthesis and Ligation of Y-Shaped Adapters}

Human fetal and adult brain cDNAs were synthesized according to the manufacturer's protocol described in the Invitrogen (Carlsbad, CA, USA) kit for cDNA synthesis, except that four degenerate oligonucleotides derived from a highly conserved region of the catalytic domain of the tyrosine kinases (PTK II) (13) (DVW-1, CCRAADGMCCABACRTCWGA; DVW-2, CCRWARCTCCAVRCRTCRCT; DVW-3, CCRAADGMCCABACRTCRCT; and DVW-4, CCHADRCTCCAGACRTCRSA; $\mathrm{R}=$ $\mathrm{A}+\mathrm{G}, \mathrm{D}=\mathrm{A}+\mathrm{T}+\mathrm{G}, \mathrm{M}=\mathrm{A}+\mathrm{C}, \mathrm{B}=$ $\mathrm{T}+\mathrm{C}+\mathrm{G}, \mathrm{W}=\mathrm{A}+\mathrm{T}, \mathrm{V}=\mathrm{A}+\mathrm{C}+\mathrm{G}, \mathrm{H}=$ $\mathrm{A}+\mathrm{T}+\mathrm{C}$, and $\mathrm{S}=\mathrm{C}+\mathrm{G}$ ) were used as primers instead of oligo-dT (Figure 1). The reaction mixture for the first-strand synthesis contained $1 \mu \mathrm{g}$ poly $(\mathrm{A})^{+} \mathrm{RNA}$ and $10 \mathrm{pmol}$ of one of the four primers, along with other components for the first-strand synthesis, except for the re- 
verse transcriptase. The mixture was incubated at $70^{\circ} \mathrm{C}$ for $10 \mathrm{~min}$ and cooled down to $50^{\circ} \mathrm{C}$ using a GeneAmp ${ }^{\circledR}$ PCR System 9600 (Applied Biosystems, Foster City, CA, USA). While the temperature was maintained at $50^{\circ} \mathrm{C}, 1 \mu \mathrm{L}$ SUPERSCRIPT ${ }^{\mathrm{TM}}$ reverse transcriptase (200 U/ $\mu \mathrm{L}$; Invitrogen) was added, and the reaction continued at $50^{\circ} \mathrm{C}$ for $1 \mathrm{~h}$. The second-strand synthesis was carried out at $16^{\circ} \mathrm{C}$ for $2 \mathrm{~h}$, and the cDNAs were then passed through Miniprep Spin Columns (Amersham Biosciences, Piscataway, NJ, USA) to remove the primers. The cDNA $(1.5 \mu \mathrm{L})$ was digested with $8 \mathrm{U} B s t$ YI or BsiHKAI (New England Biolabs, Beverly, MA, USA) for $3 \mathrm{~h}$ in a final volume of $15 \mu \mathrm{L}$. The Y-shaped adapter oligonucleotide sequences for the Bst YI digest were A1
(5'-AAATCGTAGTTATTGCTCAGACGGTCGACGGCCAG-3'), and A2 (5'-GATCCTGGCCGTCGGCTGTCTGTCGG-CGC-3'). Five hundred pmol each $\mathrm{A} 1$ and $\mathrm{A} 2$ were annealed in a total volume of $100 \mu \mathrm{L}$ annealing buffer (10 mM Tris-HCl, pH 8.0, $100 \mathrm{mM}$ $\mathrm{NaCl}$, and $1 \mathrm{mM}$ EDTA) by heating to $85^{\circ} \mathrm{C}$ and then cooling down to room temperature overnight. The Y-shaped adapters for the other restriction enzymes were made with adapter oligonucleotide pairs that formed complementary overhangs at the ends of the fragments that had been digested with the restriction enzymes. The digest (2 $\mu \mathrm{L}$ ) was ligated to $15 \mathrm{pmol}$ Y-shaped adapter in a final volume of $10 \mu \mathrm{L}$ for $16 \mathrm{~h}$ at $15^{\circ} \mathrm{C}$ using $0.1 \mu \mathrm{L}$ T4 DNA ligase (New England Biolabs).

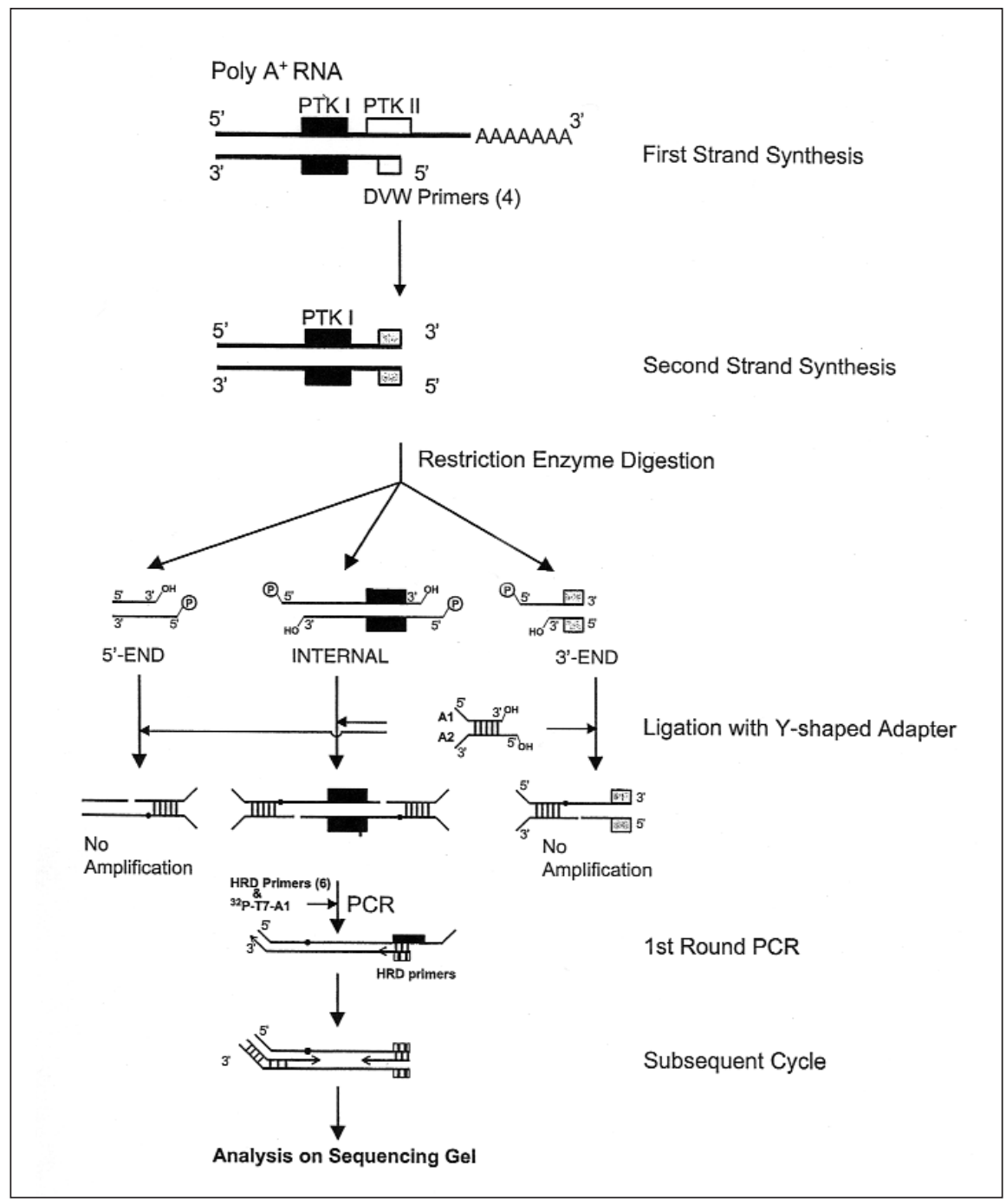

Figure 1. Schematic representation of the method for protein tyrosine kinase cDNA display.

\section{PCR and Differential cDNA Display of the Protein Tyrosine Kinase}

To amplify cDNA fragments specific to the tyrosine kinases, six kinds of degenerate oligonucleotides derived from a highly conserved region of the tyrosine kinase catalytic domain (PTK I) (13) (HRD-1, GTTSCGVGYRGCYAGGTCNCGRTG; HRD-2, GTTWCKRGCWGCYAARTCYCGRTG; HRD-3, RTTYCTDGCHGCCARGTCCCGRTG; HRD-4, GTTTCKGGCAGCMAGRTYTCTATG; HRD-5, RTTSKMRGGYTTCAMGTCTSTATG; and HRD-6, ATTRGARGGYTTSAYRTCTCTRTG; $\mathrm{S}=\mathrm{C}+\mathrm{G}, \mathrm{V}=\mathrm{A}+\mathrm{C}+\mathrm{G}$, $\mathrm{Y}=\mathrm{C}+\mathrm{T}, \mathrm{R}=\mathrm{A}+\mathrm{G}, \mathrm{N}=\mathrm{A}+\mathrm{C}+\mathrm{G}+\mathrm{T}, \mathrm{W}$ $=\mathrm{A}+\mathrm{T}, \mathrm{K}=\mathrm{T}+\mathrm{G}, \mathrm{D}=\mathrm{A}+\mathrm{T}+\mathrm{G}, \mathrm{H}=$ $\mathrm{A}+\mathrm{T}+\mathrm{C}$, and $\mathrm{M}=\mathrm{A}+\mathrm{C}$ ) were used as a 3' primer, while T7-A1 (5'-AAATCGTAGTTATTGCTCAGACGGTC- ${ }^{\prime}$ ) was used as a $5^{\prime}$ primer. To detect the fragments amplified by PCR on a display gel, 100 pmol T7-A1 was labeled at the $5^{\prime}$-end using $250 \mu \mathrm{Ci}[\gamma-32 \mathrm{P}] \mathrm{ATP}$ $\left(\mathrm{NEN}^{\circledR}\right.$ Life Science Products, Boston, MA, USA) and T4 polynucleotide kinase (New England Biolabs). After inactivating the enzyme by heating at $65^{\circ} \mathrm{C}$ for $15 \mathrm{~min}$, the labeled T7-A 1 was diluted to a final concentration of $0.31 \mu \mathrm{M}$. The PCR was carried out in a total volume of $20 \mu \mathrm{L}$ containing 10 $\mathrm{mM}$ Tris- $\mathrm{HCl}, \mathrm{pH} 8.3$, at $25^{\circ} \mathrm{C}, 50 \mathrm{mM}$ $\mathrm{KCl}, 1.5 \mathrm{mM} \mathrm{MgCl} 2,200 \mu \mathrm{M}$ dNTPs, $31 \mathrm{nM}$ labeled T7-A1, $500 \mathrm{nM}$ primer, 1 U AmpliTaq Gold ${ }^{\circledR}$ DNA polymerase (Applied Biosystems), and a $4-\mu \mathrm{L}$ aliquot of the ligation reaction mixture that had been diluted seven times with water. AmpliTaq Gold DNA polymerase was subjected to a 10 -min prePCR activation step at $95^{\circ} \mathrm{C}$, and then PCRs for the primers HRD-1 and HRD-3 were performed at $94^{\circ} \mathrm{C}$ for 30 $\mathrm{s}$ and $67^{\circ} \mathrm{C}$ for $3 \mathrm{~min}$ for 42 cycles. PCRs for the primers HRD-2, HRD-4, HRD-5, and HRD-6 were the same as described earlier, except that the annealing and extension steps were performed at $62^{\circ} \mathrm{C}$ or $57^{\circ} \mathrm{C}$ for $1 \mathrm{~min}$, respectively, and then $72^{\circ} \mathrm{C}$ for $1 \mathrm{~min}$. The PCR products from the cDNAs synthesized by DVW-1 and DVW-2 or DVW-3 and DVW-4 were combined, and $6 \mu \mathrm{L}$ of the mixture were analyzed on a $6 \%$ polyacrylamide sequencing gel. The gel was dried and exposed to 
Table 1. Classification by DNA Sequences of Bands in the Display

\begin{tabular}{|c|c|c|c|c|c|c|c|c|}
\hline Enzymes & $\begin{array}{c}\text { cDNA } \\
\text { sources }\end{array}$ & Kinases $^{a}$ & No hit'b & Knownc & Genomic & EST & $\begin{array}{l}\text { Sequencing } \\
\text { failured }\end{array}$ & Total \\
\hline \multirow[t]{2}{*}{$B s t Y I$} & Fetal & $23(15)$ & $5(4)$ & 1 & 2 & 0 & 7 & 38 \\
\hline & Adult & $27(17)$ & $6(3)$ & 1 & 0 & 0 & 5 & 39 \\
\hline BsiHKAI & Fetal & $19(14)$ & 5 & $4(3)$ & 2 & 1 & 9 & 40 \\
\hline
\end{tabular}

BioMax $^{\text {TM }}$ MR film (Eastman Kodak, Rochester, NY, USA) for $16 \mathrm{~h}$.

\section{Direct DNA Sequencing of the cDNA Fragments}

Bands were cut from the differential display gel and heated in boiling water for $5 \mathrm{~min}$, after the addition of $30 \mu \mathrm{L} 10$ $\mathrm{mM}$ Tris- $\mathrm{HCl}, \mathrm{pH} 8.0$, and $0.1 \mathrm{mM}$ EDTA. The cDNA fragments were reamplified by PCR using the Expand ${ }^{\mathrm{TM}}$ High-Fidelity PCR system (Roche Diagnostics, Indianapolis, IN, USA). The PCR products were treated with exonuclease I and shrimp alkaline phosphatase or purified on an agarose gel and subjected to DNA sequencing with an ABI PRISM ${ }^{\circledR} 377$ DNA sequencer (Applied Biosystems).

\section{Quantitative Estimation of Protein Tyrosine Kinase cDNAs in Fetal and Adult Brains}

The first-strand synthesis of the cDNA was carried out with fetal and adult brain poly(A)+ RNAs using the First-Strand cDNA Synthesis Kit for RT-PCR (Roche Diagnostics). The abundances of kinase cDNAs were measured by RT-PCR using a LightCycler ${ }^{\mathrm{TM}}$ Instrument with LightCycler ${ }^{\mathrm{TM}}$ DNA Master SYBR ${ }^{\circledR}$ Green I (both from Roche Diagnostics). The primer pairs that we used were designed from the sequences of $1000 \mathrm{bp}$ at the $3^{\prime}$-end of the kinases that is outside of the conserved regions. PCR was performed at $95^{\circ} \mathrm{C}$ for $1 \mathrm{~s}, 56^{\circ} \mathrm{C}$ for $5 \mathrm{~s}$, and $72^{\circ} \mathrm{C}$ for $10 \mathrm{~s}$. The crossing point is the RT-PCR cycle number at the point where extrapolation of the log linear region of the amplification signal crosses the baseline and correlates with the initial template concentration. Each crossing point of the RT-PCR cycle number in the kinases was corrected by assuming that the crossing point of glyceraldehyde-3-phosphate dehydrogenase (GAPDH) in both fetal and adult brain cDNAs was 17.00.

\section{RESULTS AND DISCUSSION}

We first compared the patterns of protein tyrosine kinase expression in the cDNAs of human fetal and adult brains using gene-specific degenerate and adapter oligonucleotide primers for PCR and displaying the products on a urea-polyacrylamide gel (9). However, the percentage of the bands in the display identified as coming from kinase mRNA by DNA sequence analyses was very low (less than 10\%) (data not shown). To improve specificity, we selected the mRNAs twice by applying degenerate oligonucleotide primers derived from two highly conserved regions of the catalytic domain in the tyrosine kinase. The first selection of mRNAs was carried out at the firststrand synthesis of the cDNA using the degenerate primers derived from PTK II. The resultant cDNA was digested with a restriction enzyme, followed by ligation to the Y-shaped adapter. The second selection of mRNAs was done upon PCR amplification of the cDNA fragments for the display gel using degenerate primers derived from PTK I, which is located approximately $183 \mathrm{bp}$ upstream from PTK II. Two additional factors were taken into account in designing the method for the display. One is that the number and length of the bands on the display are dependent on the frequency of the cutting sites for the restriction enzymes used. The other is that the length of the degenerate primers for the PCR amplification determines the balance of specificity and nonspecificity of the bands. In our preliminary experiments, 20-mer oligonucleotide primers produced a low frequency of specific bands, whereas 24-mer oligonucleotide primers gave a reasonably high frequency of specific bands. For these reasons, we used Bst YI and BsiHKAI for generating the site of Yshaped adapter ligation and 24-mer primers for the amplification of the cDNA fragments by PCR (Figure 1).

Figure 2 shows a differential cDNA display of the protein tyrosine kinases from human fetal and adult brains using cDNAs cut with BstYI. A total of 77 bands, 38 and 39 bands of length greater than 125 bases, were detectable from the fetal and adult mRNAs. The cDNA fragments from the bands were amplified by PCR and subjected to DNA sequence analysis and a BLAST search. Fifty bands $(65 \%)$ represented known protein tyrosine kinases, including receptor tyrosine kinases, nonreceptor tyrosine kinases, and dual-specificity/other kinases (Table 1). In some cases, there were several bands identified as coming from the same mRNA (Figure 2 , see nos. 1, 4-7, and 16) because of mispriming of the degenerate oligonucleotide primers during the first-strand synthesis from the highly expressed mRNAs. Twelve bands (15\%) failed in 
Table 2. Quantitative Estimation of the Protein Tyrosine Kinase cDNAs from the Human Fetal and Adult Brains by RT-PCR Using a LightCycler Instrument

\begin{tabular}{|lccc|}
\hline Kinases & \multicolumn{2}{c|}{$\begin{array}{c}\text { Crossing points } \\
\text { (Cycle numbers) }\end{array}$} & $\begin{array}{c}\text { Consistency } \\
\text { between RT-PCR } \\
\text { and display }\end{array}$ \\
\cline { 2 - 4 } & Fetal & Adult & no \\
EPHA4 & 23.96 & 23.64 & yes \\
EPHA5 & 21.00 & 24.38 & no \\
FGFR3 & 21.47 & 20.22 & yes \\
ERBB3 & 31.34 & 26.34 & yes \\
ERBB4 & 29.16 & 30.76 & yes \\
KIT & 30.14 & 29.76 & no \\
MET & 26.39 & 26.62 & yes \\
KDR & 28.49 & 27.25 & no \\
DDR1 & 21.02 & 22.64 & \\
First-strand synthesis of cDNAs and RT-PCR for quantitative estimation were car- \\
ried out according to the method described in the Materials and Methods section. \\
\hline
\end{tabular}

direct DNA sequencing because of poor amplification of the cDNA fragments by PCR. No significant similarities to any known genes were found in 11 bands (14\%). Six of these bands corresponded to the same cDNA fragments as determined by the DNA sequence. Two bands were found to be homologous to known genes other than kinases, Mus musculus NELF protein mRNA and Rattus norvegicus CCA3 mRNA. The net number of different tyrosine kinases was 15 and 17 bands from the fetal and adult, respectively (see parentheses in Table 1). In this experiment, the percentage of true positive bands was about $42 \%$ of the total 77 bands from the fetal and adult. The kinases identified by sequence analysis from the fetal cDNAs were EPHA8, FGFR3, and casein kinase 1 epsilon, the last of which is a serine/threonine kinase. The kinases identified in the adult cDNAs were KDR, ERBB3, MET, PDGFRB, and TYRO3.

When the cDNAs were cut with $B s i$ HKAI, a total of 63 bands were detectable in the display, and 37 or 6 bands were found to be the kinases or to show no significant similarities to known genes $(59 \%$ or $9.5 \%$, respectively). However, the net numbers of different tyrosine kinases from the fetal and adult cDNAs were 14 and 10 , respectively (see parentheses in Table 1). The percentage of true positive bands of the experiment was $38 \%$. The kinases identified in the fetal cDNAs were NTRK2,
MERTK, ERBB4, EPHA5, MAP2K5, and serine/threonine kinase KMP. The kinases identified in the adult cDNAs were FGFR3 and ERBB3. Serine/threonine kinases, KMP and casein kinase 1 epsilon, contain very similar DNA sequences in the region used for the HRD primers and were detected in the same lanes as the dual-specificity kinases.

Overall, 27 different kinases were identified in the display of the cDNAs. Because the human genome is estimated to encode 106 protein tyrosine kinase and 395 serine/threonine and mixed function kinases (12), our method displayed about $22 \%$ of this number of protein tyrosine kinases in brain cDNA using two restriction endonucleases.

To examine the validity of this approach, we compared the size of the cDNA fragments of the tyrosine kinases in the display with those predicted from the known cDNA sequence and location of restriction enzyme cutting sites and checked whether or not there were GATCT/C and A/TGCA/TC sequences for the Bst YI- and BsiHKAI-cut cDNA fragments, respectively, in the junction between the Y-shaped adapter and the cDNA fragments of the kinases. The expected cutting site and length in the display with the Bst YI digests were found in EPHA4, DDR1, JAK1, KIT, FLT1, MET, KDR, ERBB3, MAP2K5, MAP$\mathrm{K} 3$, and MAP2K4, but not in AXL, TYRO 3, EPHB4, FGFR 3, and CLK3. In the display with the BsiHKAI di- 
gests, CSF1R, DDR1, MERTK, FLT1, JAK1, ERBB4, EPHA5, ERBB3, CLK3, MAPK3, MAP2K4, and MAP2$\mathrm{K} 5$ had the predicted size, whereas NTRK2, FGFR3, and ABL1 did not. There was a discrepancy in the expression level of FGFR3 between the two displays with the Bst YI and BsiHKAI digests. However, FGFR3 had no expected cutting site for BsiHKAI or $B s t \mathrm{YI}$, indicating that the discrepancy might result from mispriming (Please note that one might be able to tell if mispriming occurred from the sequence). GATCA in place of GATCC/T at the most $5^{\prime}$ side of the cDNA fragments was found in AXL, FGFR3, and CLK3 in the display with the Bst YI digests, suggesting that star restriction cutting activity might have occurred.

To examine the validity of differences in the level of expression between the cDNAs from human fetal and adult brains seen in the display, we designed gene-specific pairs of oligonucleotide primers and carried out quantitative RTPCR with poly $(\mathrm{A})^{+}$RNAs using a

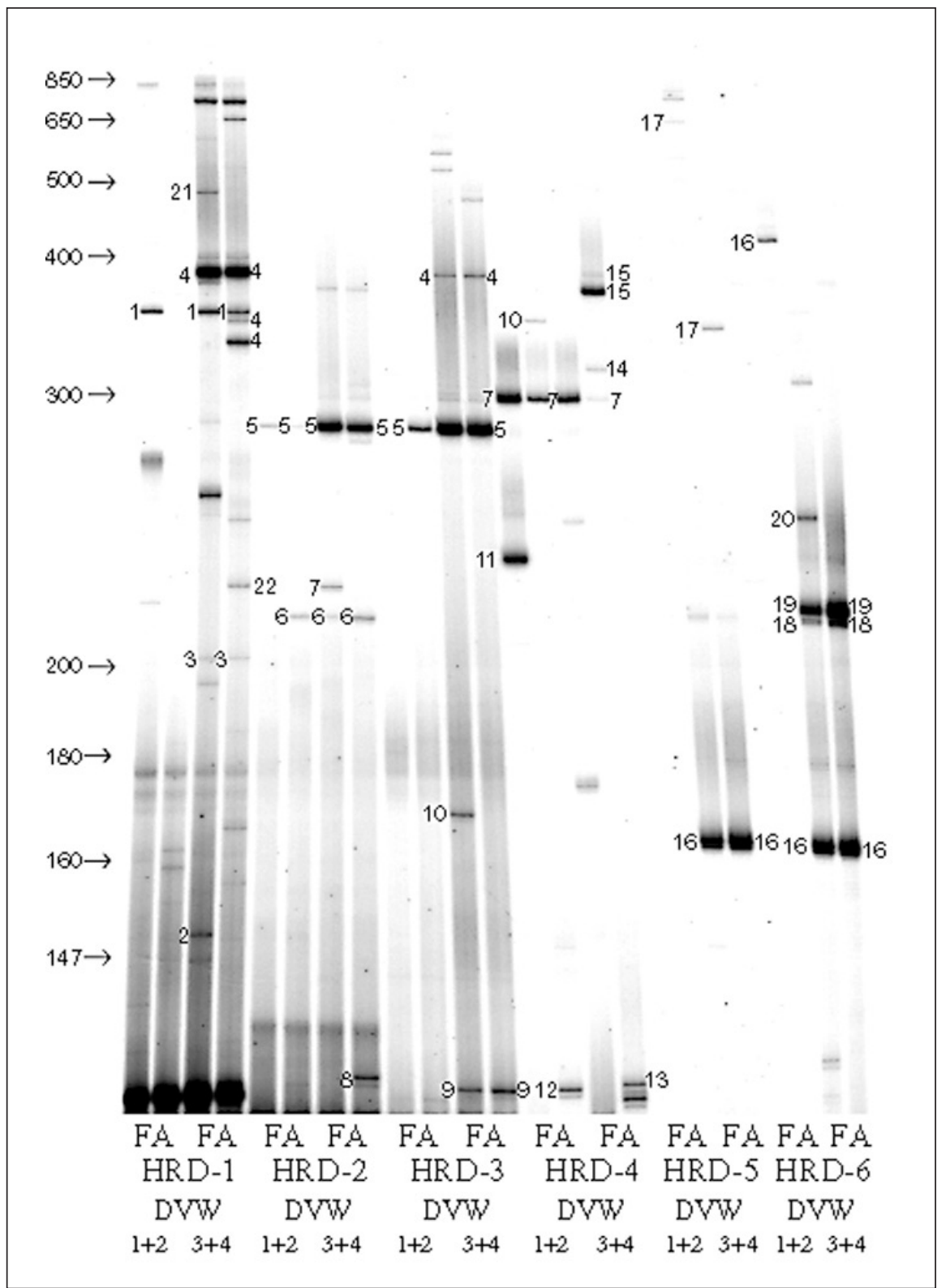

Figure 2. Differential display with the BstYI digests of the cDNAs from the fetal and adult brains. Human fetal and adult cDNAs were synthesized as described in the Materials and Methods section using four degenerate oligonucleotide primers: DVW-1, DVW-2, DVW-3, and DVW-4. After the cDNAs were digested with $B s t$ YI, they were ligated to the Y-shaped adapter, and the cDNA fragments were then PCRamplified between the T7-A1 labeled with ${ }^{32} \mathrm{P}$ and six degenerate oligonucleotide primers: HRD-1, HRD-2, HRD-3, HRD-4, HRD-5, and HRD-6, respectively. The PCR products were analyzed on a 6\% polyacrylamide gel containing $8 \mathrm{M}$ urea. Lanes HRD-1, HRD-2, HRD-3, HRD-4, HRD-5, and HRD-6 indicate bands amplified by the primers, HRD-1, HRD-2, HRD-3, HRD-4, HRD-5, and HRD-6, respectively. Lanes DVW 1+2 and DVW 3+4 indicate the bands from the cDNAs, the first strand of which was synthesized by the primers, DVW-1 and DVW-2 and DVW-3 and DVW-4, respectively. Lanes F and A indicate the bands from the fetal cDNA or the adult cDNA, respectively. The numbers with arrows indicate molecular standards expressed as bases. The numbers indicate known genes: 1, ephrin receptor EphA4 (EPHA4); 2, ephrin receptor EphA8 (EPHA8); 3, megakaryocyte-associated tyrosine kinase (MATK); 4, discoidin domain receptor family, member1 (DDR1); 5, Janus kinase 1 (JAK1); 6, c-kit oncogene (KIT); 7, AXL receptor tyrosine kinase (AXL); 8, TYRO3 protein tyrosine kinase (TYRO3); 9, fms-related tyrosine kinase 1 (FLT1); 10, ephrin receptor EphB4 (EPHB4); 11, fibroblast growth factor receptor 3 (FGFR3); 12, met proto-oncogene (MET); 13, kinase insert domain receptor (KDR); 14, platelet-derived growth factor receptor $\beta$ polypeptide (PDGFRB); 15 , epidermal growth factor receptor 3 (ERBB3); 16, mitogen-activated protein kinase 5 (MAP2K5); 17, CDC-like kinase 3 (CLK3); 18, mitogen-activated protein kinase 3 (MAPK3); 19, mitogen-activated protein kinase 4 (MAP2K4); 20, casein kinase 1 epsilon; 21, Mus musculus NELF protein; and 22, Rattus norvegicus CCA3. 
LightCycler (Table 2). ERBB4, EPHA5, and DDR1 were expressed at significantly higher levels in the fetal cDNA than in the adult in RT-PCR, although DDR1 was expressed at the same level in both fetal and adult cDNAs in the display. The expression levels of FGFR3, ERBB3, and KDR were significantly increased in the adult cDNA in RT-PCR. EPHA4, KIT, and MET were expressed at the same level in both cDNAs in RTPCR, although MET was found only in the adult cDNA in the display. The inconsistency in the quantitative estimation of the kinases between the display and RT-PCR was mainly observed in the high level of expression of the kinases, probably because of mispriming. In summary, the expression levels of the protein tyrosine kinases in both fetal and adult cDNAs estimated by RT-PCR using the LightCycler and seen in the display were comparable to each other in most cases, except for the highly expressed mRNAs.

Recent progress in the microarray method $(2,3)$ makes it simpler than other methods $(7,9)$, including SAGE (11), for the analysis of expression patterns of known genes. However, our method offers a convenient approach to identify which tyrosine kinases are present and therefore which are potential targets for real time RT-PCR. The method avoids cloning and screening of cDNA fragments and yields larger fragments and a larger number of kinases from each restriction digest than previous display methods. The method also allows the detection of even low-abundance tyrosine kinases and is applicable to a range of organisms, including those without expressed sequence tag (EST) catalogues, sequenced genomes, or microarray availability. The positions of the bands corresponding to known kinases are generally reproducible from gel to gel, and the approach has the potential to discover new tyrosine kinases, especially in the unsequenced organisms.

\section{ACKNOWLEDGMENTS}

This research was supported by grants from the Ministry of Education, Science, Sports, and Culture of Japan to Y.Y.

\section{REFERENCES}

1.Albright, T.D., T.M. Jessell, E.R. Kandel, and M.I. Posner. 2000. Neural science: a century of progress and the mysteries that remain. Cell 100:S1-S55.

2.Chee, M., R. Yang, E. Hubbell, A. Berno, X.C. Huang, D. Winkler, J. Lockhart, M.S. Morris et al. 1996. Accessing genetic information with high-density DNA arrays. Science 274:610-614.

3.DeRisi, J., L. Penland, P.O. Brown, M.L. Bittner, P.S. Meltzer, M. Ray, Y. Chen, Y.A. Su et al. 1996. Use of a cDNA microarray to analyze gene expression patterns in human cancer. Nat. Genet. 14:457-460.

4.Drescher, U., F. Bonhoeffer, and B.K. Muller. 1997. The Eph family in retinal axon guidance. Curr. Opin. Neurobiol. 7:175-180.

5.Hanks, S.K. and R.A. Lindberg. 1991. Use of degenerate oligonucleotide probes to identify clones that encode protein kinases. Methods Enzymol. 200:525-532.

6.Kung, H.-J., H.-C. Chen, and D. Robinson. 1998. Molecular profiling of tyrosine kinases in normal and cancer cells. J. Biomed. Sci. 5:74-78.

7.Liang, P. and A.B. Pardee. 1992. Differential display of eukaryotic messenger RNA by means of the polymerase chain reaction. Science 257:967-971.

8.Lindberg, R.A. and E.B. Pasquale. 1991. Isolation of cDNA clones that encode active protein-tyrosine kinases using antibodies against phosphotyrosine. Methods Enzymol. 200:557-564.

9.Prashar, Y. and S.M. Weissman. 1996. Analysis of differential gene expression by display of $3^{\prime}$ end restriction fragments of cDNAs. Proc. Natl. Acad. Sci. USA 93:659663.

10.Robinson, D., F. He, T. Pretlow, and H.-J. Kung. 1996. A tyrosine kinase profile of prostate carcinoma. Proc. Natl. Acad. Sci. USA 93:5958-5962.

11.Velculescu, V.E., L. Zhang, B. Vogelstein, and K.W. Kinzler. 1995. Serial analysis of gene expression. Science 270:484-487.

12.Venter, J.C., M.D. Adams, E.W. Myers, P.W. Li, R.J. Mural, C.G. Sutton, H.O. Smith, M. Yandell et al. 2001. The sequence of the human genome. Science 291:13041351.

13.Wilks, A.F. 1989. Two putative protein-tyrosine kinases identified by application of the polymerase chain reaction. Proc. Natl. Acad. Sci. USA 86:1603-1607.

Received 3 July 2001; accepted 10 December 2001.

\section{Address correspondence to:}

Dr. Yukio Yasukochi

Department of Molecular Genetics

Medical Research Institute

Tokyo Medical and Dental University

Tokyo 113-8510, Japan

e-mail: yyasmgen@mri.tmd.ac.jp 